Suzuki(1), Tadashi Yamamoto(3), Atsushi Nakagawa(1). (Laboratory of Supramolecular Crystallography, Institute of Protein Research, Osaka Univ; 2:Medical Top Track program Medical Research Institute, Tokyo Medical and Dental University; 3:Division of Oncology, Institute of Medical Science, Univ of Tokyo)

Ubiquitination plays an important role in many basic cellular events, and it controlled by a multi-enzyme cascade that involves E1, E2, and E3 enzyme activities. The $\mathrm{Cbl}$ (Casitas $\mathrm{B}$-lineage lymphoma) family proteins, $\mathrm{Cbl}, \mathrm{Cbl}-\mathrm{b}$, and $\mathrm{Cbl}-\mathrm{c}$, are thought to negatively regulate the signaling through protein-tyrosine kinases as ubiquitin E3 ligases. The $\mathrm{Cbl} / \mathrm{Cbl}-\mathrm{b}$ bind to specific sequence motifs containing phosphotyrosine (D/N-x-pY-(S/T)-x-X- or -D-pY-R-) of protein-tyrosine kinases, but Cbl-c only binds to $-(\mathrm{D} / \mathrm{N})-\mathrm{x}-\mathrm{pY}-(\mathrm{S} / \mathrm{T})-\mathrm{x}-\mathrm{x}-$ motif This substrate binding specificity based on their structures is still unclear. In this study, we determined the crystal structure of the Cbl-c TKB domain at 1.6 angstrom resolution. The structure of Cbl-c free-form was similar to that of $\mathrm{Cb}$ closed-form in the substrate-bound state. Differences of the free-form structure of $\mathrm{Cbl}$ and $\mathrm{Cbl}-\mathrm{c}$ may result in the specific substrate binding strategy. In addition, we determined the crystal structure of Cbl-c mutant, which had a mutation at a hinge-like residue possibly critical for open/close hinge motion of the Cbl-c upon substrate binding, at 2.4 angstrom resolution. Indeed, this Cbl-c mutant showed an open-form structure which was similar to the $\mathrm{Cbl}$ free-form. Therefore, we expect that the substrate specificity of this Cbl-c hinge-like residue mutant turns to that of $\mathrm{Cbl} / \mathrm{Cbl}-\mathrm{b}$ type. We are now performing the biochemical assay to evaluate the binding property of $\mathrm{Cbl}, \mathrm{Cbl}-\mathrm{c}$ and their mutants.

\section{P-058 未構造タンパク質の機能と進化的特性の解析}

Analyses of Functional and Evolutionary Characteristics of Disordered Proteins

Huang He (1),Akinori Sarai (1). (1:Dept Life bioscience and Bioinformatics, Graduate School of Computer Science and Systems Engineering, Kyushu Institute of Technology)

Disordered regions of proteins have been found to play important role in various biological functions. Thus, it would be interesting to examine the relationship among sequence, interactions, network, evolution, expression and disease etc. associated with disordered proteins. In this study, we analyzed functional and evolutionary characteristics of disordered proteins in various genomes. We calculated the $\mathrm{Ka} / \mathrm{Ks}$ value (ratio of non-synonymous and synonymous mutation rates) for both the disordered and structured regions, and found that disordered proteins are under stronger positive selection than structured proteins. Also, disordered regions are under less functional constrains.Furthermore, we investigated the correlation between expression level and disorder/structure region. Our result shows that more disordered regions are expressed at low level than structured regions, whereas less disordered regions expressed at high level than structured regions. Previous studies showed that many regions with low leve expression play a very important role of forming new exon or new domain.Mor in-depth studies are now ongoing: such as the formation of disorder region in the phylogenetic view and the relation between disorder regions and particular human disease. Using the Treefam database and ORI-GENE tool, we found that some disordered regions are specific in human, Further investigation will be necessary to test whether these regions/proteins may are related to the formation of some human traits.

\section{P-059 膜変形タンパク質ドメインの分子ダイナミクス}

Molecular Dynamics of the Membrane-Deforming Protein Domain

Kazuhiro Takemura(1,2), Shiro Suetsugu(1,3) and Akio Kitao(1,4)(1:Institute of Molecular and Cellular Biosciences, University of Tokyo, 2:Institute for Molecular Science, 3:PRESTO,JST, 4:JST,CREST)

The membrane-deforming protein domains participate in essential biological processes, such as endocytosis and the formation of lamellipodia or filopodia. The representative of protein domains above is Bin-amphiphysin-Rvs (BAR) domain, which forms the crescent-shaped dimer. Its concave surface is postulated to induce membrane deformation of a specific curvature and to cause long tubulation of the artificial liposomes. In contrast to the BAR domain, the Rac binding (RCB) domain forms a "zeppelin-shaped" dimer and is considered to induce inward tubulation of the artificial liposomes (in a direction opposite to that induced by the BAR domain, thus the RCB domain is also called the inverse-BAR domain). Mutagenesis experiments and biophysical analysis specified the amino acids responsible for membrane deformation and suggested that the convex surface of the RCB domain dimer has essential role, however, the detailed mechanism is not fully understood.

In order to investigate the atomistic details of membrane deformation induced by the RCB domain, we performed molecular dynamics simulation. The X-ray structure of IRSp53 RCB domain (1wdz.pdb) and lipid bilayer (DOPC/DOPE/DOPS $=4: 4: 1$ ) were placed in a periodically repeating box filled with water molecules with $0.1 \mathrm{M} \mathrm{KCl}$. We will report how the RCB domain dimer behaves in solution and interacts with lipid membranes.

\section{P-060 好冷性酵素と中温性酵素における構造柔軟性と触媒活性}

Structual flexibility and catalytic activity in psychrophilic and mesophilic enzymes

Takahiro Kosugi (1) and Shigehiko Hayashi (1). (1: Department of Chemistry, Graduate School of Science, Kyoto University)

It is known that some enzymes exhibit peculiar temperature dependence. In particular, psychrophilic enzymes are significantly more active at low temperatures compared to homologous mesophilic ones in spite of close similarity of the amino acids constituting the active sites. Although it is often considered that the catalytic activity at low temperature is related to structural flexibility of the enzyme, it is still not clear how the flexibility affects the activity. Moreover, it is not known which enzymatic step is relevant to the temperature dependence. It may be binding of the substrate, making or breaking of the chemical bonds or unbinding of the product. At the first stage of our research, we compared the response (change of fluctuation) upon binding with the substrate in psychrophilic and mesophilic $\alpha$-amylases. We calculated the difference of root mean square fluctuations and entropies between free and complex states of the enzymes with molecular dynamics simulation. Quasiharmonic approximation was used to calculate the entropies. By dividing the entropies into contributions of each residue, we found the remarkable difference of entropies in the active site between psychrophilic and mesophilic $\alpha$-amylases. It is suggested that the structural flexibility that alters the entropy in the active site is responsible for the temperature dependence of enzyme.

\section{P-061 マウス $\alpha$ デフェンシン, cryptdin4 の殺微生物作用の分子機構} Mechanism of bactericidal activity of mouse $\alpha$-defensin cryptdin 4

Koji Masuda, Naoki Sakai and Tokiyoshi Ayabe (Department of Cellular Life Science, Faculty of Advanced Life Science, Hokkaido University)

In mammal, three antimicrobial peptides subfamilies, $\alpha$-defensins, $\beta$-defensins and $\theta$-defensins are expressed leukocytes, mucosal surfaces, skin and other epithelia. These peptides can kill directly a variety of pathogens. Defensin family peptides have the cationic net charge, and this property is the advantage to interact with bacterial cell surface. The difference of $\alpha$ - and $\beta$-defensins is disulfide bond pairs of their conserved six cysteines. In mouse, $\alpha$-defensins named cryptdins are expressed in Paneth cells at the base of crypts in small intestine and are secreted on to the crypt lumen in response to the bacterial stimulation. Among cryptdins, the bactericidal activity of cryptdin 4 is known to be most potent.In this study, to elucidate the molecular mechanism of the bactericidal activity of cryptdin 4 of mouse small intestine, we prepared the chemically synthesized cryptdin 4 . The synthetic cryptdin 4 were reduced form, so disulfide bond should be introduced into the peptide. Disulfide bond formation in the peptide by dimethyl sufoxide (DMSO) was carried out. Refolded peptides were purified by RP-HPLC, and evaluated disulfide formation and folding by MALDI-MS and Acid-Urea PAGE. The bactericidal activities against defensin sensitive $S$. typhimurium of refolded peptides were almost same as wild type. We established a method to prepare the biologically active cyptdin 4 . Further, we report data of the structure-function relationship with bactericidal function of cryptdin 4 .

2P-062 カタニン $\mathrm{p} 60$ による微小管切断メカニズムの構造生物学的基盘

Structural basis of mechanisms on microtubule severing by Katanin $\mathrm{p} 60$

Yoshie Fujiwara (1), Yohta Kuwahara (1,3), Naoko Iwaya (1,2), Kohei Akiyama (3). Natsuko Goda (1) and Hidekazu Hiroaki (1,3). (1: Div. Struct. Biol, Graduate School of Medicine, Kobe Univ; 2: Div. Molecular Design, Graduate School of Engineering, Kyoto Univ; 3: Int. Graduate School of Arts and Sciences, Yokohama City Univ)

Katanin p60 (Kp60), a type I AAA-ATPase family member consisting of one conserved AAA domain, is a key enzyme involved in microtubule disassembly and severing. We have focused on identifying a novel domain from the $\mathrm{N}$-terminal regions of Kp60 (Kp60 NTD), since most of the other AAA-ATPases possess their unique N-terminal domains with specific adaptor and / or substrate binding activities. Indeed, we confirmed that Kp60 NTD had tubulin binding activity in vitro. Recently, we have succeeded to solve its solution structure by NMR, after optimizing the domain boundary by using a rational domain-boundary optimization strategy. The structure of Kp60 NTD has comprised three anti-parallel a-helices, strikingly resembling to the other MIT domains. The sequence identity between Kp60 NTD and MIT domains is rather low, and thus, the alanine zipper, a proposed interhelical motif of the MIT domain, has not been found in the Kp60 NTD structure. The substrate binding surface was determined by amino acid substitution experiments. The tubulin binding interface was mapped on the third helix of Kp60 NTD. The interface is again strikingly similar to that of the MIT domain of Vps4, which recognizes ESCRT-III complex on the late-endosomal membrane and promotes invagination of the multivesicular membrane. ESCRT-III complex is known as the late-endosome specific membrane skeleton. Our structural study of Kp60 NTD reveals the common molecular mechanisms underlying disassembly of cytoskeleton and membrane skeleton.

\section{P-063 ストラクチュロームでの分子ネットワークの解析}

Analysis of Biomolecular Network in Structurome

Mitsuaki Ohtsuaka (1), Satoshi Fujii (1), Akinori Sarai (1)(1:Dept, Bioscience and Bioinformatics, Kyushu Institute of Technology

Most of biological functions are achieved by the cooperative action of huge number of molecules in the network. In order to understand the molecular mechanism of the cooperative action in the molecular network and to develop drugs, structural information of molecules and their interactions are essential. However, the size of biomolecular structural space (structurome) is expanding very rapidly, and the structurome is becoming more complex, as it is populated by many molecular complexes in recent years. In order to extract useful information from the structurome, we have developed a database/tool of biomolecular network, PDBnet, based on the structural information of PDB (Protein Data Bank). PDBnet enables users to extract various kinds of information on the network of molecular clusters and to visualize the network. By using this tool, we have analyzed the relationship among various molecular properties such as the size of molecules, structural and physicochemical characteristics of interaction regions, and the network properties such as the number of possible interaction partners, in a 
systematic way. We have also compared the molecular properties with evolutionary information and essentiality of proteins within the context of network. We will present recent results of these analyses.

\section{P-064 Streptococcus sobrinus グルコシルトランスフェラーゼー| のデ キストラン結合に伴う熱容量変化}

Heat capacity change for the binding of Streptococcus sobrinus glucosyltransferase-I to dextran

Hideyuki Komatsu (Dept Bioscience \& Bioinformatics, Graduate School of Computer Science \& Systems Engineering, Kyushu Institute of Technology)

Glucosyltransferase-I (GTF-I) secreted by mutans streptococcus catalyzes the transfer of glucosyl units from the cleavage of sucrose to a growing glucan chain. Sucrose $+(\text { Glucose })_{n} \rightarrow$ Fructose $+(\text { Glucose })_{n+1}$

GTF-I $(1.75 \mathrm{kDa})$ is composed of two functional domains: an N-terminal catalytic domain (GSd, $110 \mathrm{kDa}$, GS for glucansucrase) and a C-terminal glucan-binding domain (GBd, $65 \mathrm{kDa}$ ). The binding of the GBds to dextran $(\alpha(1-6)$ glucan) was previously studied by using isothermal titration calorimetry (ITC) to determine the changes of Gibbs energy, enthalpy, and entropy ( $\Delta G, \Delta H$ and $\Delta S)[1]$. The result at $25^{\circ} \mathrm{C}$ demonstrates that the strong binding $(\Delta G=-44 \mathrm{~kJ}$ $\left.\mathrm{mol}^{-1}\right)$ is driven by negatively large enthalpy $\left(\Delta H=-289 \mathrm{~kJ} \mathrm{~mol}^{-1}\right)$ accompanied with large entropy loss $\left(-T \Delta S=245 \mathrm{~kJ} \mathrm{~mol}^{-1}\right)$

Herein, we reports the heat capacity change $\left(\Delta C_{p}\right)$ of binding, which parameter provides information about the changes in solvation and conformation of interacting molecules. ITC was used to determine the $\Delta H$ for the binding of GTF-I or its truncated proteins to dextran. Temperature-dependent $\Delta H$ was then measured, from which $\Delta C_{p}$ was calculated. The preliminarily estimated value was largely negative, suggesting release of large amount of hydrophobic hydration water. In this presentation, we will report more accurate $\triangle C p$, and discuss the changes in solvation and conformation of the protein and dextran upon their binding.

[1] Komatsu et al. Biochemistry (2007) 46, 8436-8444.

\section{P-065 タンパク贒内環境下における新しいpKa の第一原理計算による提 案}

Theoretical proposal of a new pKa concept applicable in protein environment by the first principles calculations.

Tomonori Tanaka (1), Katsumasa Kamiya (2), Yasuteru Shigeta (2) and Kenji Shiraishi(1). (1:Graduate School of Applied Physics, Univ of Tsukuba; 2:Institute for Picobiology, Graduate School of Life Science, Univ of Hyogo)

It is important to understand the behavior of protons in biomolecules for clarifying mechanisms of the physiological functions at the atomic level. However, accurate determination of the positions of protons and/or protonation states is still difficult for high-molecular-weight biomolecules such as membrane proteins. In such circumstances, $\mathrm{pKa}$ is commonly used to obtain information about protonation states of amino acid residues participating in physiological functions. Yet it should be noted that $\mathrm{pKa}$ is a thermodynamical parameter, defined originally for aqueous solution. The behavior of protons in protein environment is different entirely from that in aqueous solution in a sense that proton dynamics is much more constrained in the protein by its three-dimensional structure, suggesting that the use of the $\mathrm{pKa}$ definition in aqueous solution is inadequate for estimations of $\mathrm{pKa}$ in protein environment. Therefore, it is imperative to extend the $\mathrm{pKa}$ concept for the acquisition of the precise proton behavior under protein environment. In this study, we propose a new $\mathrm{pKa}$ concept, which is applicable in protein environment and compatible with the conventional $\mathrm{pKa}$, on the basis of the first-principles calculations combined with the metadynamics approach that provide the precise estimations of free energies.

\section{P-066 リボヌクレアーゼPタンパク質 Rpp21.とRpp29 の複合体形成が触 媒活性に重要である}

The complex formation of Rpp21 and Rpp29 plays an important role in catalytic activity of ribonuclease $\mathrm{P}$

Takashi Honda (1), Yoshimitu Kakuta (1,2) and Makoto Kimura (1,2). (1: Dept System Life Sciences, 2: Dept. Bioscience and Biotechnology, Graduate School, Kyushu University)

Ribonuclease $\mathrm{P}$ (RNase $\mathrm{P}$ ) is a ribonucleoprotein complex involved in the processing of the 5'-leader sequence of precursor tRNA (pre-tRNA). The human RNase P protein subunits Rpp21 and Rpp29, which bind to each other, with a catalytic RNA (H1 RNA) are sufficient for activating endonucleolytic cleavage of pre-tRNA. Here we have determined the crystal structure of the complex between the Pyrococcus horikoshii RNase P proteins PhoRpp21 and PhoRpp29, the archaeal homologs of Rpp21 and Rpp29, respectively. PhoRpp21 and PhoRpp29 form a heterodimeric structure, where the N-terminal two helices $(\alpha 1$ and $\alpha 2)$ in PhoRpp21 predominantly interact with the N-terminal extended structure, the $\beta$-strand $(\beta 2)$, and the C-terminal helix $(\alpha 3)$ in $P h o R p p 29$. The interface is dominated by hydrogen bonds and several salt bridges, rather than hydrophobic interactions. The electrostatic potential on the surface of the heterodimer shows a positive charged cluster on one face, suggesting a possible RNA binding surface of the PhoRpp21-PhoRpp29 complex. The present structure along with the mutational analysis suggests that the heterodimerization of PhoRpp21 and PhoRpp29 plays an important role in function of $P$. horikoshii RNase P.

2P-067 細菌べん毛フックキャップの結晶構造

Crystal Structure of Salmonella Flagellar Hook Cap, a Scaffolding Protein for
Hook Assembly

Hideyuki Matsunami(1,2), Katsumi Imada(1,3), Keiichi Namba(1,3).(1:Okinawa Institute of Science and Technology;2:Dynamic NanoMachine, ICORP, JST;3:Graduate School of Frontier Biosciences, Osaka Univ)

The bacterial flagellum is a large macromolecular complex composed of the filament, the hook and the basal body. Three capping proteins, FliD, FlgD and FlgJ, are required for assembly of the filament, the hook and the rod, respectively. FlgD is essential for polymerization of FlgE to form the hook; FlgE is excreted to the cell exterior without polymerization in the absence of FlgD, suggesting that FlgD forms the hook cap to facilitate the folding of exported FlgE in unfolded forms and its incorporation into the growing end of the hook. To understand the mechanism of the hook cap for hook assembly, structural information of FlgD is essential. Here we report a crystal structure of Salmonella FlgD in its pentameric form. Diffraction data were obtained at the SPring-8 beamline BL41XU and the structure was determined at 3.6- $\AA$ resolution. The electron density map revealed a "mushroom-shaped" structure of the FlgD pentamer. The N-terminal half of FlgD forms the stalk of the mushroom as a bundle of $\alpha$-helices, although unambiguous model building is rather difficult due to relatively low resolution of the map of this portion. A flexible conformation of this region might play an important role in the hook capping mechanism. The C-terminal half, which is better resolved, forms the cap portion of the mushroom with a diameter of $90 \AA$, approximately a half of the hook. We will discuss possible mechanisms of the FlgD cap for efficient hook assembly as well as the similarities and differences between the three caps for flagellar assembly.

2P-068 Dynamics of amino acids in the electron transfer pathways between 0xygenase and Ferredoxin components in Rieske oxygenase system

Dynamics of amino acids in the electron transfer pathways between Oxygenase and Ferredoxin components in Rieske oxygenase system

Wayne Dawson (1), Tohru Terada (2) and Kentaro Shimizu (1). (1: Department of Biotechnology; 2: Agricultural Bioinformatics Research Unit, Graduate School of Agricultural and Life Sciences, The University of Tokyo)

Oxygeneses $(\mathrm{Ox})$ are involved in many metabolic pathways associated with the degradation of aromatic hydrocarbons; typically to detoxify them. Ferredoxin (Fd) is notable for its role in such processes as photosynthesis. Both contain a non-heme $\mathrm{Fe}_{2} \mathrm{~S}_{2}$ that can be used to transfer electrons. Here we examine refinements of the $\mathrm{X}$-ray structure of carbazole 1,9a-dioxygenase (CARDO) using MD simulation to understand the stability and dynamics of the amino acids; particularly those that are likely to permit electron transfer between the $\mathrm{Ox}$ and $\mathrm{Fd}$ subunits. The structure consists of three subunits of Fd:Ox forming. We find in this study that the general structure of CARDO remains stable during the simulation with a maximum rmsd of approximately $2 \AA\left(\right.$ all $\left.\mathrm{C}_{\alpha} / \mathrm{C}_{\beta}\right)$ from the observed X-ray structure. In addition, the main residues that are thought to be located along the electron transfer path appear to interact with each other regularly during the $5 \mathrm{~ns}$ simulation permitting a proton conduction pathway.

\section{P-069 モルフォロジーを利用した蛋白質表面の様々な深さのポケットを} 同時に計算するアルゴリズムの開発

A fast algorithm for detection of pockets with multi-scale depth on protein surface by mathematical morphology

Takeshi Kawabata(1). (1. Grad.Sch.Info.Sci., NAIST)

Detection of pockets on protein surfaces is an important step to find putative binding sites of small molecules. Last year, we proposed a novel algorithm using small and large probe spheres (Kawabata and Go, Proteins, 68:516,2007); the pockets is defined as the region where the small sphere can enter, but a large sphere cannot. The radius of large probe is considered as the "depth" of pockets. However, a depth of binding pockets is widely distributed, and each type of binding molecule has a specific preference for the pocket depth; coenzymes like deep pockets, but peptides/oligosaccharides like shallow ones. If a type of binding molecule is unknown, we have to search for pockets with various depth values. In this study, we invented an efficient pocket detection algorithm for multi-scale probe pockets, which simultaneously calculate pockets with several different depths. The algorithm is based on the mathematical morphology for the 3D grids of proteins and probes, which have been applied for the protein pockets by Masuya and Doi (J.Math.Graph.13:331, 1995). The efficient multi-scale algorithm is constructed by the inclusion relationship of pockets: pockets for smaller spheres are parts of those for larger spheres. Using this algorithm, we can quickly calculate shallowness of pockets, Rinaccess (minimum radius of inaccessible probe spheres) for the protein residue and atoms, which provides clues to predict types of binding molecules.

2P-070 Effect of kosmotropic and chaotropic solutes on a allosteric protein under non-denaturing conditions

Effect of kosmotropic and chaotropic solutes on a allosteric protein under non-denaturing conditions

Antonio Tsunieshige (1) and Yuuki Yashiro (2): (I: Dept of Frontier Biosciences, Faculty of Biosciences and Applied Chemistry, Hosei University; 2: Materials Science, Faculty of Engineering, Hosei University)

We have studied the impact of several kosmotropic as well as chaotropic solutes under non-denaturing conditions on the allosteric function of human hemoglobin ( $\mathrm{HbA})$, namely, oxygen affinity for the first (K1) and last (K4) oxygenation steps, 